\title{
Radiological Downstaging with Neoadjuvant Therapy in Unresectable Gall Bladder Cancer Cases
}

\author{
Sushma Agrawal*, Lalit Mohan, Chandan Mourya, Zafar Neyaz, Rajan Saxena
}

\begin{abstract}
Background: Gall bladder cancer (GBC) usually presents as unresectable or metastatic disease. We conducted a feasibility study to evaluate the effect of neoadjuvant therapy (NAT) on radiologic downstaging and resectability in unresectable GBC cases. Materials and Methods: Patients with locally advanced disease were treated with chemoradiotherapy [CTRT] ( external radiotherapy (45Gy) along with weekly concurrent cisplatin 35mg/ $\mathrm{m}^{2}$ and 5-FU $500 \mathrm{mg}$ ) and those with positive paraaortic nodes were treated with neoadjuvant chemotherapy [NACT (cisplatin $25 \mathrm{mg} / \mathrm{m}^{2}$ and gemcitabine $1 \mathrm{gm} / \mathrm{m}^{2}$ day 1 and 8,3 weekly for 3 cycles). Radiological assessment was according to RECIST criteria by evaluating downstaging of liver involvement and lymphadenopathy into complete response (CR), partial response (PR), stable disease (SD) and progressive disease (PD). Results: A total of 40 patients were evaluated from January 2012 to December 2014 (CTRT=25, NACT=15). Pretreatment CT scans revealed involvement of hilum (19), liver infiltration (38), duodenum involvement ( $n=22)$, colon involvement $(n=11)$, N1 involvement $(n=11)$, N2 disease $(n=8)$, paraaortic $L N(n=15)$, and no lymphadenopathy $(n=6)$. After neoadjuvant therapy, liver involvement showed CR in 11(30\%), PR in $4(10.5 \%)$, SD in $15(39.4 \%)$ and lymph node involvement showed CR in $17(50 \%)$, PR in $6(17.6 \%)$, SD in $4(11.7 \%)$. Six patients (CTRT=2, NACT=4) with $66.6 \%$ and $83 \%$ downstaging of liver and lymphnodes respectively underwent extended cholecystectomy. There was $16.6 \%$ and $83.3 \%$ rates of histopathological CR of liver and lymph nodes. All resections were Ro. Conclusions: Neoadjuvant therapy in unresectable gall bladder cancer results in a $15 \%$ resectability rate. This approach has a strong potential in achieving $\mathrm{R0}$ and node negative disease. Radiologic downstaging $(\mathrm{CR}+\mathrm{PR})$ of liver involvement is $40.5 \%$ and lymphadenopathy is $67.5 \%$. Nodal regression could serve as a predictor of response to neoadjuvant therapy.
\end{abstract}

Keywords: Radiologic downstaging - neoadjuvant therapy - unresectable gallbladder cancer

Asian Pac J Cancer Prev, 17 (4), 2137-2140

\section{Introduction}

Gall bladder cancer is endemic in northern India and often presents as locally advanced or metastatic disease (Randi et al., 2006). Complete surgical resection is the only treatment modality with curative potential. It has a median survival of 9 months after simple cholecystectomy, and 19 months after extended cholecystectomy in patients who are apparently resectable. The clinical diagnosis of gallbladder cancer is challenging due to the lack of specific signs and symptoms and consequently, the diagnosis is often not made until the disease is unresectable. Down-staging these tumours by neoadjuvant therapy could possibly render these tumours resectable and allow standard surgery to be carried out, as has been attempted and demonstrated by few investigators (Aretxabala et al.,1999; Kato et al., 2013).

Unresectable GBC are those which are involving the portal vein, hepatic artery, common bile duct,adjacent organs and those with distant nodal disease or metastases. The proximity of the gallbladder neck to the right portal pedicle and right hepatic duct poses limitations on the surgical margins in this area without compromising the integrity of the right liver - these tumors more often than not, end up receiving only palliation (Saxena et al., 1995).The preoperative identification of gross bulky hilar lymphadenopathy is a strong relative contraindication to surgery.

Given the very poor prognosis of gallbladder cancer in the setting of bulky radiologically evident adenopathy, some investigators have advocated preoperative therapy in order to downstage and render these patients resectable. The earliest published phase II report by Arexabala et al.(1999) reported $86 \%$ resectability rate with neoadjuvant 5 -fluorouracil and radiation in 18 potentially resectable gall bladder cancers. At a median follow-up of 24 months, $46 \%$ were alive. Amongst those who died only one patient had a local recurrence. This kind of optimistic response in a disease like gall bladder cancer, with nihilistic five year survival figures overall, merits addressing de-novo gall bladder cancers at primary stage rather than as incidental gall bladder cancers, which are biologically early lesions

Department of Radiotherapy, Sanjay Gandhi Post Graduate Institute of Medical Sciences, Lucknow, India *For correspondence: sushmaagrawal@yahoo.co.uk 
as compared to locally advanced gall bladder cancers. The other published study of neoadjuvant chemotherapy in locally advanced GBC from Japan demonstrated 36.5\% resectability rate (Kato et al., 2013).

We report radiologic downstaging in a cohort of unresectable GBC patients who received neoadjuvant therapy as a part of our prospective feasibility study.

\section{Materials and Methods}

This is a prospective feasibility cohort study approved by institute ethics committee. Patients with GBC, proven cytologically or histologically with TNM Stages IIIa, IIIb, IVa were included in this study. The inclusion criteria for patients was involvement of right branch of the hepatic artery or portal vein or common bile duct, common hepatic duct and primary biliary confluence or liver, duodenum and colon involvement and lymphadenopathy in hepatoduodenal, peri-pancreatic, common hepatic artery regions (N1). Jaundiced patients were to be included in this trial only after endoscopic palliation of jaundice. Patients with recognizable metastasis (M1) and having following criteria: age $>70$ years, untreated surgical obstructive jaundice (SOJ), ascites, history of prior chemotherapy for locally advanced or metastatic disease, uncontrolled co-morbidities (like diabetes mellitus, coronary artery disease, chronic renal failure, chronic lung disease) making them unfit for chemoradiotherapy or anesthesia and major surgical undertaking, ECOG performance status $>1$, Platelets $<100.000 \times 10^{9} / \mathrm{L}$, serum creatinine $>1.5 \mathrm{X}$ institutional upper limit of normal (IULN), serum bilirubin $>1.5 \mathrm{X}$ IULN, serum ALT $>2.5 \mathrm{X}$ IULN, history of weight loss $>15 \%$, inadequate dietary intake (at least 1500 cals/ day) were excluded from this study. After an year patient recruitment was below the expectations and patients more often presented with enlarged paraaortic lymphnodes, hence the inclusion criteria was relaxed to include these patients with advanced disease as well.

\section{Primary objective}

To assess the radiological downstaging of locally advanced carcinoma gallbladder with neoadjuvant therapy as per RECIST criteria (Therasse et al., 2009).

\section{Treatment schedule}

Patients underwent basic diagnostic investigations (complete history, physical examination, triple phase CT scan abdomen, chest X-ray, routine hematology, and serum chemistry and FNAC). A diagnostic laparoscopy to rule out presence of disseminated disease was desirable in all patients. In jaundiced patients, laparoscopy was performed after palliation of jaundice, prior to induction into study.

For patients undergoing chemoradiation a treatment planning scan was done on Somatom Sensation Open CT scanner (M/s Siemens Medical System, Germany) and the data was imported into Eclipse planning system. Radiotherapy was delivered by a linear accelerator to gallbladder tumour and enlarged lymphnodes with 1.5 $\mathrm{cm}$ margin using at least $6 \mathrm{MV}$ photons or a combination with $15 \mathrm{MV}$ photons by 3DCRT technique upto a dose of 45Gy/25\#/5weeks along with concurrent chemotherapy consisting of weekly injection 5-Fluorouracil $325 \mathrm{mg} /$ $\mathrm{m}^{2}$ and injection cisplatinum $35 \mathrm{mg} / \mathrm{m}^{2}$ with adequate hydration and antiemetic cover. Prophylactic irradiation of unenlarged periportal, peripancreatic, retroportal, coeliac, superior mesenteric and paraaortic lymphnodes upto L2 vertebral level upto 45 Gy was also mandatory. For those presenting with enlarged or suspicious coeliac, superior mesenteric or paraaortic lymph nodes (N2 or more), neoadjuvant chemotherapy (NACT) was administered for 3 cycles (cisplatin $25 \mathrm{mg} / \mathrm{m}^{2}$ and gemcitabine $1 \mathrm{gm} / \mathrm{m}^{2}$ day 1 and 8, 3 weekly). If patients had adequate downstaging after 3 cycles they were subjected to surgery and if not, then further 3 cycles were given before a restaging triple phase CT scan was done for evaluation for resectability. Weekly toxicity assessment using NCI-CTC criteria for haematological and non-haematological toxicity during chemoradiation or chemotherapy was also done (ctc version 2003).

\section{Evaluation of efficacy of treatment}

Radiological assessment of disease status prior to neoadjuvant treatment was as per the AJCC 7th edition (AJCC 7th Edition Cancer Staging Manual). A triple phase CT scan 4-6 weeks after completion of treatment (chemoradiation or 3-6 cycles chemotherapy) was done to evaluate radiological downstaging. Radiological downstaging was graded into complete response (CR), partial response (PR), stable disease (SD) and progressive disease (PD) according to Response Evaluation Criteria in Solid Tumours (RECIST) for extent of liver infiltration and lymph node size. Indentation or loss of fat plane with duodenum and colon was considered involvement of these structures and complete restoration of fat plane was considered as complete response, partial resolution as partial response, no resolution as stable disease and progression of infiltration was considered as progressive disease. Irregularity of the vessel wall or narrowing was considered as vessel involvement. Resolution of vessel wall abnormality or restoration of its patency was considered as response to neoadjuvant therapy.

\section{Surgery}

All patients showing radiological signs of resectability 4 weeks after completion of CTRT or NACT were considered for surgical resection. The surgical procedure were classified as microscopically radical (R0), macroscopically complete but microscopically non-radical (R1) or macroscopically incomplete (R2).

\section{Follow-up}

Patients with R0 disease were kept only on follow-up and those with R1 disease were offered postoperative radiotherapy (in those who received neoadjuvant chemotherapy). Thereafter patients were followed up every 3 months for 2 year and then every 6 months until local progression, death, loss to follow-up or until 36 months after study inclusion in radiation oncology and surgical gastroenterology OPD.

\section{Statistical considerations}

A sample size of 50 was taken assuming 10-20\% 
resectability rate after chemoradiation.This is an interim analysis of radiological downstaging in 40 patients with this approach. Conversion of radiological downstaging to resectability rate and conversion of radiological response to pathological response is also being reported. Treatment compliance, morbidity, mortality and survival will be reported separately after adequate follow-up. Analysis of the study was according to intent to treat.

\section{Results}

Forty patients have been inducted into this study from January 2012 to December 2014.The median age of patients was 52 years (interquartile range 35-57 years), male: female ratio is 9:31.Only $50 \%$ patients underwent diagnostic laparoscopy due to logistic reasons.19 presented with obstructive jaundice and underwent stenting prior to neoadjuvant therapy. $7.5 \%(n=3)$ patients progressed on treatment and $15 \%(n=6)$ deteriorated on or after completion of treatment and hence 2 sets of CT scans for response assessment were available for 31 patients only. $62.5 \%$ patients $(n=25)$ were treated by CTRT and $37.5 \%$ $(n=15)$ by NACT. $10 \%$ patients with obstructive jaundice treated with CTRT were radiologically suitable for further surgical evaluation, but succumbed to gastrointestinal bleeding after completion of treatment.

\section{Radiological downstaging}

The pretreatment CT scans revealed involvement of hilum in $47.5 \%(n=19)$, liver infiltration (any) in $95 \%(\mathrm{n}=38)$, liver infiltration $>2 \mathrm{~cm}$ in $70 \%(\mathrm{n}=28)$, duodenum involvement in $55 \%(\mathrm{n}=22)$, colon involvement in $27.5 \%(\mathrm{n}=11), \mathrm{N} 1$ station involvement (periportal lymphadenopathy) in $27.5 \%(n=11), \mathrm{N} 2$ disease (coeliac and superior mesenteric lymphadenopathy) in $20 \%$ $(n=8)$, paraaortic lymphadenopathy in $37.5 \%(n=15)$, no lymphadenopathy in $15 \%(n=6)$. Right hepatic artery was involved in $30 \%(n=12)$, common hepatic artery was involved in $12 \%(\mathrm{n}=5)$, portal vein was involved in $25 \%(n=10)$.
After neoadjuvant therapy $(\mathrm{CTRT}=25, \mathrm{NACT}=15)$, the overall radiological response rate $(\mathrm{CR}+\mathrm{PR})$ was 40.5 $\%$ in any liver involvement, $24.9 \%$ in liver infiltration $>2 \mathrm{~cm}, 76.8 \%$ in duodenum and $62.5 \%$ in colon (Table 1). There was $36.3 \%$ partial response in 22 patients who presented with vessel involvement (any). Radiological resolution of hepatic artery or portal vein involvement was seen in $25 \%$ and $12.5 \%$ patients and the rest had static disease. Out of 34 patients who presented with lymphadenopathy $67.6 \%$ showed overall radiological response (CR+PR). $36.3 \%$ with $\mathrm{N} 1$ lymphadenopathy, $62.5 \%$ with N2 lymphadenopathy and $53.3 \%$ with paraaortic lymphadenopathy revealed radiological CR (Table 2).

\section{Resectability rate}

6 patients underwent extended cholecystectomy $(\mathrm{CTRT}=2, \mathrm{NACT}=4)$.All resections were $\mathrm{R} 0$. All resected patients had preoperative liver infiltration $(n=6), 2$ had colon involvement and 3 had duodenal wall abutment. All had lymphadenopathy $(\mathrm{N} 1=1, \mathrm{~N} 2=3$, paraaortic $\mathrm{LN}=2)$. Four patients (66.6\%) showed radiological CR at primary but the histopathological CR was seen in one patient only

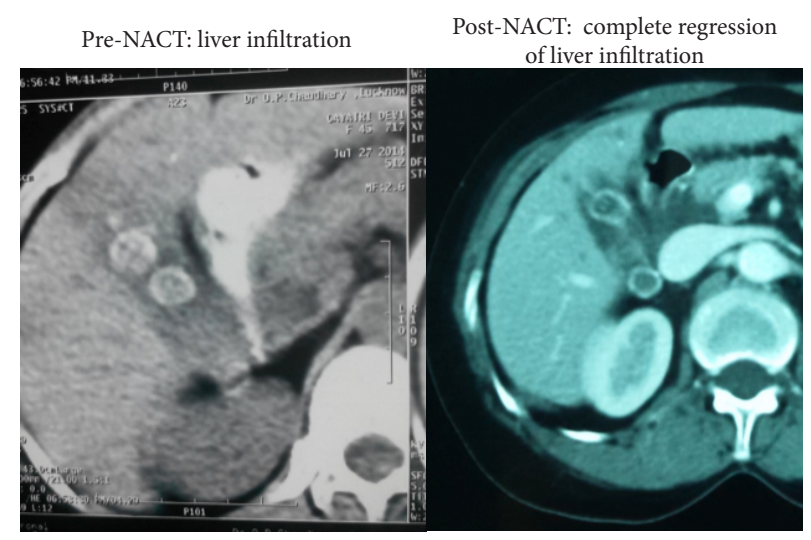

Figure 1. Illustration of Radiological CR Liver Infiltration, on Histopathology: pathological CR of Gallbladder and Liver

Table 1. Radiological Downstaging of Liver, Colon, Duodenum and Vessels

\begin{tabular}{|c|c|c|c|c|c|}
\hline & $\begin{array}{c}\text { Radiological } \\
\text { CR } \%(\mathrm{n})\end{array}$ & $\begin{array}{c}\text { Radiological PR } \\
\%(\mathrm{n})\end{array}$ & $\begin{array}{c}\text { Radiological } \\
\text { Static disease } \\
\%(\mathrm{n})\end{array}$ & $\begin{array}{l}\text { Radiological } \\
\text { progressive } \\
\text { disease } \%(\mathrm{n})\end{array}$ & $\begin{array}{c}\text { Not evaluable } \\
\%(\mathrm{n})\end{array}$ \\
\hline Liver infiltration $(n=38,95 \%)$ & $30(11)$ & $10.5(4)$ & $39.4(15)$ & - & $21(8)$ \\
\hline Liver infiltration $>2 \mathrm{~cm}(\mathrm{n}=28,70 \%)$ & $21.4(6)$ & $3.5(1)$ & $50(14)$ & - & $25(7)$ \\
\hline Duodenum $(\mathrm{n}=22,55 \%)$ & $45.4(10)$ & $31.8(7)$ & $2.5(1)$ & - & $18.1(4)$ \\
\hline Colon $(n=12,30 \%)$ & $54.5(6)$ & $8(1)$ & $16.6(2)$ & - & $25(3)$ \\
\hline Arterial involvement $*(n=12,30 \%)$ & - & $25(3)$ & $75(9)$ & - & - \\
\hline Venous involvement $(\mathrm{n}=10,25 \%)$ & - & $12.5(5)$ & $12.5(5)$ & - & - \\
\hline
\end{tabular}

*Involvement of common hepatic artery and right hepatic artery clubbed together.

Table 2. Radiological Downstaging of Various Stations of Lymph Nodes

\begin{tabular}{|c|c|c|c|c|c|}
\hline & $\begin{array}{l}\text { Radiological } \\
\text { CR \%(n) }\end{array}$ & $\begin{array}{l}\text { Radiological } \\
\text { PR } \%(n)\end{array}$ & $\begin{array}{l}\text { Radiological } \\
\text { Static } \%(n)\end{array}$ & $\begin{array}{l}\text { Radiological } \\
\text { progressive } \\
\text { disease } \%(\mathrm{n})\end{array}$ & Not evaluable \%(n) \\
\hline $\mathrm{N} 1(\mathrm{n}=11,27.5 \%)$ & $36.3(4)$ & $36.3(4)$ & $4(1)$ & 0 & $8(2)$ \\
\hline $\mathrm{N} 2(\mathrm{n}=8,20 \%)$ & $62.5 \%(5)$ & $12.5(1)$ & 0 & 0 & 2 \\
\hline Paraaortic $(\mathrm{n}=15,37.5 \%)$ & $8(53.3)$ & $1(6.6)$ & $3(20 \%)$ & $1(6.6 \%)$ & $13.3(2)$ \\
\hline Overall & $17(50 \%)$ & $6(17.6 \%)$ & $4(11.7 \%)$ & $1(2.9 \%)$ & $\begin{array}{c}12(30 \%) \text { (6 had no upfront } \\
\text { lymphadenopathy) }\end{array}$ \\
\hline
\end{tabular}




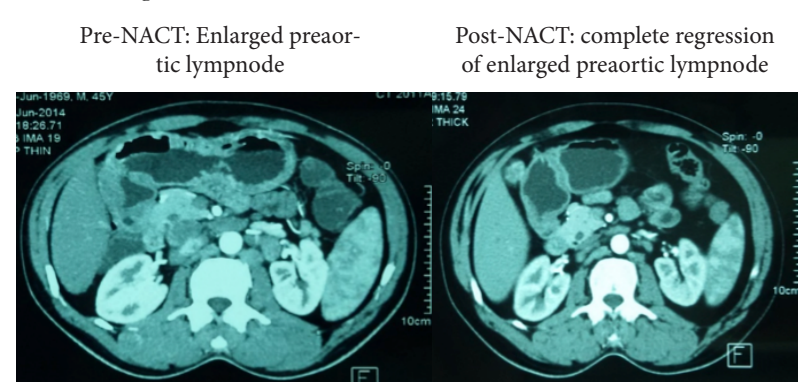

Figure 2. Illustration of Radiological CR Preaortic Lympnode, Ro Resection, on Histopathology: Pathological CR of Lymphnode

(16.6\%) (Figure 1). 83.3\% (5/6) showed radiological CR at lymphnodes and all had histopathological CR (Figure 2). At a median follow-up of 18 months, four out of 6 patients are alive till date. One patient expired in the immediate postoperative period and the other developed liver metastases after a 24 months disease free period and succumbed after a course of secondline chemotherapy.

\section{Discussion}

To the best of our knowledge this is the first detailed report on radiologic response assessment to neoadjuvant therapy in unresectable gall bladder cancer. Neoadjuvant therapy results in high overall radiological response rate $(\mathrm{CR}+\mathrm{PR})$ in these patients. It is $40.5 \%$ in patients with any liver involvement, $24.9 \%$ in liver infiltration $>2 \mathrm{~cm}$, $76.8 \%$ in duodenum and $62.5 \%$ in colon involvement. Some $30 \%$ of patients with any liver involvement and $20 \%$ with liver infiltration $>2 \mathrm{~cm}$ achieved radiological $\mathrm{CR}$, this also being the case in $45 \%$ of patients with duodenum involvement and $54 \%$ with colon involvement. The histopathological CR at primary site was $2.5 \%$.The conversion rate of radiological CR to histopathological $\mathrm{CR}$ for liver infiltration was $9 \%$.

Similarly radiological response rates in downstaging of lymphadenopathy is also very encouraging. The overall radiological response rate is $67.6 \%$ for lymphadenopathy at any site. $72.6 \%$ of patients with $\mathrm{N} 1$ disease, $75 \%$ with $\mathrm{N} 2$ and $59.9 \%$ with paraaortic lymphadenopathy. $36.3 \%$ with N1 lymphadenopathy, $62.5 \%$ with N2 lymphadenopathy and $53.3 \%$ with paraaortic lymphadenopathy showed radiological CR. The histopathological CR was $12.5 \%$.The conversion rate of radiological CR to histopathological $\mathrm{CR}$ is $29.4 \%$ in this series and is very promising for unresectable GBC.

Aretxabala reported 55.5\% pathologic CR in liver and lympnodes in a potentially resectable subset of GBC who underwent neoadjuvant chemoradiation (Aretxabala et al., 1999). Their study included patients with disease limited to gallbladder bed and N1 station lymphadenopathy while in our study patients had N1, N2 and paraaortic lymphadenopathy. Kato reported $13.6 \%$ PR and 50\% SD with neoadjuvant gemcitabine in a series of 22 unresectable GBC patients and the pathologic CR rate for GB and liver was not seen while it was $22 \%$ for lymphadenopathy and this is comparable to our series (Kato et al., 2013). Radiological resolution of vascular involvement was seen in only a quarter of patients and none of them were rendered resectable. Similar results were observed in the Japanese series (Kato et al., 2013).

Neoadjuvant therapy in our series resulted in $15 \%$ resectability rate, $36.3 \%$ in Japanese series and $57.4 \%$ and $47 \%$ in Indian series of 122 and 17 patients respectively (Sirohi et al., 2012; Gangopadhyay et al., 2015). All resected patients in our series had R0 resection, while $18 \%$ in Japanese series underwent $\mathrm{R} 0$ resection and 18\% underwent R1 resection (Kato et al., 2013). Our low resectability rates could be explained by disease progression or morbidity in $22.5 \%$ patients presenting with obstructive jaundice.

There is no known predictor of response to chemotherapy in GBC. The results of this study demonstrate that lympnode regression with neoadjuvant therapy is a good predictor of histopathological response.

The limitations of this study were non uniform protocol of treatment due to low accrual and the compliance for diagnostic laparoscopy was 50\% due to logistic reasons.

In conclusion, neoadjuvant therapy in unresectable gall bladder cancer results in $15 \%$ resectability rate. This approach has a strong potential in achieving $\mathrm{R} 0$ and node negative disease. Radiologic downstaging $(\mathrm{CR}+\mathrm{PR})$ of liver involvement is $40.5 \%$ and lymphadenopathy is $67.5 \%$. Nodal regression could serve as a predictor of response to neoadjuvant therapy.

\section{References}

ctep.cancer.gov/protocolDevelopment/electronic.../docs/ ctcaev3.pdf

de Aretxabala X, Roa I, Burgos L, et al (1999). Preoperative chemoradiotherapy in the treatment of gallbladder cancer, Am Surg, 65, 241-6.

https://cancerstaging.org/.../AJCC6th Ed Cancer Staging Manual Part1.pdf

Gangopadhyay A, Nath P, Biswas J (2015). Reduced dose intensity of chemotherapy may not lead to inferior palliation in locally advanced carcinoma of the gall bladder: An experience from a regional cancer center in Eastern India, $J$ Gastrointest Cancer, 46, 297-300

Kato A, Shimizu H, Ohtsuka M, et al (2013). Surgical resection after downsizing chemotherapy for initially unresectable locally advanced biliary tract cancer: a retrospective singlecenter study, Ann Surgical Oncol, 20, 318-24.

Randi G, Franceschi S, La Vecchia C (2006). Gallbladder cancer worldwide:geographical distribution and risk factors, Int $J$ Cancer, 118, 1591-2.

Saxena R, Sikora SS, Kaushik SP (1995). A triple bypass procedure for advanced carcinoma of the neck of the gall bladder. Br J Surg, 82, 394-5.

Sirohi B, Mitra A, Jagannath P, et al (2015). Neoadjuvant chemotherapy in patients with locally advanced gallbladder cancer. Future Oncol, 11, 1501-9.

Therasse P, Arbuck SG, Eisenhauer EA, Kaplan RS, Rubinstein L (2000). New guidelines to evaluate the response to treatment in Solid Tumors. J Natl Cancer Inst, 9, 205-6. 